\title{
PEDAGOGÍA HOSPITALARIA. UNA ASIGNATURA PENDIENTE EN LA FORMACIÓN UNIVERSITARIA DE LOS PROFESIONALES DE LA EDUCACIÓN
}

\author{
HOSPITAL PEDAGOGY. AN UNFINISHED BUSINESS IN THE \\ UNIVERSITY TRAINING OF EDUCATION PROFESSIONALS
}

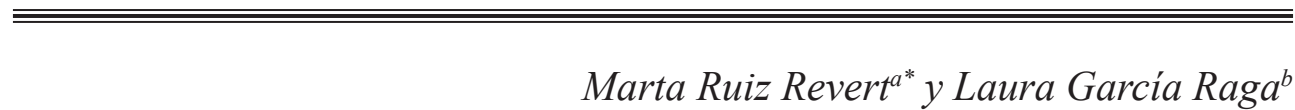

Fechas de recepción y aceptación: 29 de octubre de 2018, 27 de febrero de 2019

Resumen: La pedagogía hospitalaria se desarrolla donde hay alumnado enfermo, velando por el derecho a la educación de los niños y las niñas. Ello implica que la formación en pedagogía hospitalaria no debe estar dirigida únicamente a los docentes hospitalarios, ya que cualquier docente puede, a lo largo de su carrera profesional, atender en sus aulas a niños/as que enfermen. Este artículo tiene como objetivo reflexionar sobre la necesidad de dicha formación, justificándola a partir de una investigación que ha supuesto el análisis e interpretación de diferentes datos, documentos legislativos y planes de estudio. Para ello, se utiliza una metodología cualitativa, haciendo uso del análisis documental como instrumento. Concluimos nuestro trabajo poniendo de manifiesto la escasez de acciones formativas dentro de estudios universitarios que doten de unas competencias específicas en pedagogía hospitalaria a los futuros maestros y las cuales deberían dirigirse a favorecer la atención integral del paciente pediátrico.

Palabras clave: derecho a la educación, necesidad de formación, profesión docente, pedagogía hospitalaria.

Abstract: Hospital Pedagogy is developed where there is an ill student body, watching over the children's right to an education. It implies that the formation in Hospital Pedago-

${ }^{a}$ Facultad de Educación. Universidad Cardenal Herrera CEU.

* Correspondencia: Universidad CEU Cardenal Herrera. Calle Carmelitas, 3. 03203 (Elche), Alicante. España.

E-mail: marta.ruiz@uchceu.es

${ }^{\mathrm{b}}$ Facultad de Filosofía y Ciencias de la Educación. Universidad de Valencia. 
gy must not only address the hospital teachers and schoolteachers, since any teacher can, throughout their professional career, assist children that become ill in their classrooms. This article has as an aim to think about the need for such formation, drawing on a pierce of research that has supposed the analysis and interpretation of different data, legislative documents and plans of study. In order to do so, a qualitative methodology is being used, putting into practice the documentary analysis as an instrument. We conclude our work by revealing the shortage of formative actions inside university studies that provide few specific competences in Hospital Pedagogy for future teachers and that such teachers should favour the integral attention of paediatric patients.

Keywords: right to education, need of formation, educational profession, hospital pedagogy.

\section{INTRODUCCIÓN}

La educación implementa el desarrollo de las personas y es un derecho fundamental del que la enfermedad no debe privar a quien la padece. Y es que, tal y como afirman Torralba et al. (2006), la educación es un instrumento que puede paliar la vulnerabilidad de quienes se encuentran en una situación inerme o, en su defecto, ayudar al otro a aceptar su vulnerabilidad y conseguir el mayor grado de autonomía posible.

Por todo ello, la defensa del derecho a la educación de niños enfermos y/u hospitalizados es un tema de creciente interés para muchos profesionales de la educación. Ahora bien, aunque son muchos los avances que las investigaciones en materia de pedagogía hospitalaria han aportado a la disciplina y son ya muchos los países que cuentan con normativas referentes a la atención educativa de alumnado enfermo y/u hospitalizado (Lizasoáin, 2016b), sigue existiendo un desconocimiento social de esta realidad y muy pocos profesionales de la educación conocen la forma en la que esta atención se materializa. Y es que, tal y como se presenta en el artículo que nos ocupa, son muy escasas las universidades del ámbito nacional las que contemplan en sus planes de estudio asignaturas relacionadas con la pedagogía hospitalaria.

De acuerdo con Riquelme et al. (2013), citado en Violant (2015a), proponer que la pedagogía hospitalaria pase a formar parte de la formación universitaria de los futuros maestros es una medida que puede favorecer tanto la mejora de la atención educativa de los menores enfermos como la producción 
científica en este campo de estudio, ya que es la universidad el lugar desde donde se puede reivindicar y paliar esta necesidad emergente. En esta línea, Anguera y Violant afirman que

la Pedagogía Hospitalaria, como disciplina, necesita, por un lado, un mayor número de investigadores que publiquen los resultados de sus estudios, sobre todo los que se encuentran en la práctica (atención educativa en el hospital, en la escuela o en el domicilio), y por otro lado un mayor número de tesis doctorales que configuren a esta disciplina como cuerpo de conocimiento (2015: 151).

Cabe destacar que la atención educativa de los niños hospitalizados no recae exclusivamente en los maestros hospitalarios, sino que es una tarea compartida entre el tutor del menor y el docente hospitalario. No se debe obviar que cuando un menor ha de someterse a un ingreso hospitalario que le impide la asistencia regular a su centro de referencia aquel sigue siendo alumno de su colegio y, por tanto, cualquier docente puede, a lo largo de su carrera profesional, atender en sus aulas a alumnos que deban someterse a un ingreso hospitalario.

En todo este contexto, resulta necesario profundizar en la temática y conocer determinados datos que ponen en evidencia la necesidad de acercar la pedagogía hospitalaria a cuantos profesionales de la educación desarrollan su carrera profesional en el ámbito educativo. En concreto, las preguntas a las que se da respuesta en el presente artículo, enmarcado en una investigación amplia que se desarrolló a modo de tesis doctoral, son las siguientes: ¿qué volumen de ingresos hospitalarios de niños/as menores de 18 años se producen en España anualmente?, ¿cuál es la estancia media de los ingresos?, ¿qué perfil profesional se ocupa de la atención educativa en las unidades pedagógicas hospitalarias?, ¿cómo se accede a los puestos de maestro/a hospitalario/a?, ¿contemplan las titulaciones de Pedagogía, Educación Social y Magisterio de las universidades del ámbito nacional la formación en pedagogía hospitalaria? Y más importante todavía, ¿debe estar enfocada la formación en pedagogía hospitalaria exclusivamente a los/las profesionales de la educación que trabajan en el contexto hospitalario? 


\section{OBjetivos}

Para responder a las preguntas anteriormente planteadas, se propone como objetivo general reflexionar sobre la necesidad de que la pedagogía hospitalaria forme parte de la formación de los profesionales de la educación como asignatura en los planes de estudio universitarios del ámbito nacional. Para la consecución del citado objetivo, se proponen los siguientes objetivos específicos:

- Analizar los datos de morbilidad hospitalaria en España de niños y jóvenes entre 0 y 24 años durante el año 2016.

- Realizar una aproximación a la regulación de la atención educativa de los menores enfermos en España.

- Presentar la prevalencia de la pedagogía hospitalaria en los actuales planes de estudio.

\section{Metodología}

Atendiendo al criterio de datación o a la naturaleza de los datos analizados, para la consecución de los objetivos expuestos se ha partido de un enfoque interpretativo, utilizando una metodología cualitativa cuyo instrumento principal de recogida de datos ha sido el análisis documental. En concreto, considerando el primer objetivo específico, centrado en conocer el estado actual de la morbilidad hospitalaria en España, se han analizado e interpretado los datos estadísticos relativos al número de ingresos de menores en nuestro país y las estancias medias para el año $2016^{1}$.

En relación con el segundo objetivo específico, se ha realizado una revisión bibliográfica centrada en la regulación de la atención educativa de los menores enfermos en España, donde se ha analizado exhaustivamente toda la legislación existente desde el año 1982, momento en el que aparece en España la primera normativa que recogió y legisló la necesidad de atender educati-

\footnotetext{
${ }^{1}$ El Instituto Nacional de Estadística publica en noviembre de cada año los datos referentes al año anterior; por ello, a fecha de octubre de 2018, estaban publicados los datos del año 2016.
} 
vamente a los niños que eran ingresados en los hospitales del ámbito nacional hasta la actualidad. Los datos que aportamos en el apartado de resultados harán referencia a la legislación vigente por motivos obvios de extensión.

Así mismo, se ha utilizado el análisis documental como técnica de recogida de datos para analizar la prevalencia de la pedagogía hospitalaria en los actuales planes de estudio de las titulaciones de Magisterio, Pedagogía y Educación Social de todas las universidades públicas, privadas y a distancia del ámbito nacional. Cabe indicar que, en la investigación, los documentos analizados atienden a la clasificación de "material oficial" y han abarcado para la consecución del tercer objetivo específico todos los planes de estudio de las mencionadas titulaciones. El procedimiento seguido para obtener esta información ha sido minucioso y ha consistido en extraer, de la página del Ministerio de Educación, Cultura y Deporte, tres listados que reflejan las universidades que imparten cada uno de los grados analizados. Posteriormente, se ha accedido a la página de cada universidad y se han revisado, uno por uno, 122 planes de estudio.

\section{ANÁLisis E INTERPRETACión DE RESULTAdos}

Con referencia a los resultados de los análisis realizados, se pasan a exponer en este punto los datos, siguiendo el orden de los objetivos de la investigación propuestos en el artículo que nos ocupa.

\subsection{Morbilidad hospitalaria en España}

Para dar respuesta al primer objetivo específico planteado en el presente artículo se presentan los datos de morbilidad hospitalaria en España. Estos datos permiten conocer el número de ingresos hospitalarios por grupo de edad producidos en un año, así como su duración media. El análisis de los datos permite contextualizar la investigación y poner de manifiesto una realidad que merece ser tenida en cuenta, ya que atender desde el ámbito educativo a los alumnos enfermos contribuye a la mejora de su calidad de vida (Violant, Molina y Pastor, 2011). 
En la tabla 1 se presentan datos del número de ingresos hospitalarios de niños y jóvenes de 0 a 24 años en España durante el año 2016, así como las estancias medias hospitalarias.

TABLA 1

Estancia media de los ingresos hospitalarios en España según la edad del paciente en niños/as y jóvenes entre 0 y 24 años en el año 2016

\begin{tabular}{|l|c|c|c|c|}
\hline & $\leq 1$ año & $1-4$ años & $5-14$ años & $15-24$ años \\
\hline de 1 a 30 días & 5,45 & 3,48 & 3,45 & 3,80 \\
total ingresos & 135.560 & 102.413 & 132.858 & 188.182 \\
\hline de 31 días a 6 meses & 62,15 & 57,75 & 55,35 & 61,47 \\
total ingresos & 4.933 & 613 & 1.194 & 3.164 \\
\hline de 6 meses a 1 año & 244,98 & 328,30 & 234,11 & 262,93 \\
total ingresos & 12 & 7 & 15 & 216 \\
\hline + de 1 año & 0 & 454 & 0 & 446,06 \\
total ingresos & 0 & 2 & 0 & 66 \\
\hline
\end{tabular}

Fuente: elaboración propia a partir de los datos consultados en el Instituto Nacional de Estadística (2018).

\subsection{Regulación de la atención educativa de los menores enfermos en la legislación nacional y autonómica de la Comunitat Valenciana}

La normativización y regulación de la atención educativa de los menores hospitalizados en España son relativamente recientes. Autores como González, Macías y García (2002) datan los inicios de la atención educativa "efectiva" en los hospitales en 1964. Con respecto a la formación exigida a los maestros que desarrollan su carrera profesional en el contexto hospitalario, como se indica en algunos trabajos, Ruiz (2016: 169) asegura que "la legislación educativa vigente en nuestro país no exige a los profesionales que desarrollan su trabajo en las Unidades Pedagógicas Hospitalarias una formación previa específica en el ámbito de la Pedagogía Hospitalaria”. Los procedimientos de acceso a las vacantes docentes en las Unidades de Pedagogía Hospitalaria son diferentes entre unas comunidades autónomas y otras, pudiendo acceder a ellas mediante comisiones de servicios, concursos de traslados o incluso a 
través de contrataciones realizadas directamente por el Ministerio de Sanidad y Consumo (Guillén y Mejía, 2002, y Molina y Violant, 2009, citados en Ruiz, 2016).

En el caso concreto de la Comunitat Valenciana, existen actualmente tres documentos que establecen los criterios y procedimientos para la atención educativa durante la hospitalización, así como la regulación de la atención domiciliaria. La Orden de 4 de julio de 2001, de la Consellería de Cultura y Educación, por la que se regula la atención al alumnado con necesidades de compensación educativa, especifica en el apartado segundo que o bien los/as niños/as están sometidos/as a hospitalizaciones de larga estancia o bien los menores usuarios de atención domiciliaria serán considerados/as, a todos los efectos, alumnos/as con necesidades de compensación educativa.

Por otro lado, la Orden de 21 de noviembre de 2006 de la Consellería de Cultura, Educación y Deporte, por la que determinan los criterios y procedimientos para la atención hospitalaria y domiciliaria del alumnado que requiera compensación educativa en Educación Primaria y Secundaria obligatoria se establece el objeto de las unidades educativas hospitalarias, los recursos personales de dichas unidades, así como la jornada laboral del profesorado que las atiende. Tal y como reflejan otros trabajos, "en ningún momento se hace referencia en este documento a las competencias específicas de formación que deberían poseer los docentes que trabajan en ellas" (Ruiz, 2016: 70).

Para finalizar con la normativización de la atención educativa en el contexto hospitalario y domiciliario, la instrucción de 18 de agosto de 2017, de la Dirección general de política educativa por la que se regula el funcionamiento de las Unidades Pedagógicas Hospitalarias ubicadas en hospitales públicos de la Comunidad Valenciana para el curso 2017/2018, especifica que esta atención se desarrollará en las quince unidades pedagógicas hospitalarias ubicadas en quince centros hospitalarios públicos de la citada comunidad.

Retomando el perfil profesional del maestro hospitalario, se destacan las aportaciones de Lizasoáin y Lieutenant (2002), quienes ponen de manifiesto la necesidad de que los profesionales de la educación que desarrollan su trabajo en el contexto hospitalario posean una formación específica en pedagogía hospitalaria. Ello es motivado, entre otras muchas causas, por el elevado número de pacientes atendidos, la angustia que sufren los familiares de los 
pacientes y la necesidad de atender a los enfermos desde un enfoque multidisciplinar.

En este sentido, Ruiz (2016), de acuerdo con Lizasoáin y Lieutenant (2002), destaca que la formación en pedagogía hospitalaria es necesaria por múltiples razones, entre las que se destaca el contexto en el que se desarrolla la labor docente, ya que

La preparación psicológica y mental para hacer frente a situaciones tan complejas como estas, al objeto de saber gestionarlas, es asimismo importante, al igual que las propias competencias profesionalizadoras más especificas. La relación ámbito educativo-escenario familiar es, en estos casos, todavía más decisiva que en situaciones de normalidad; la preparación competencial para ello es, en consecuencia, un aspecto básico y fundamental (p. 325).

\subsection{Prevalencia de la pedagogía hospitalaria en los planes de estudio de las titulaciones de Educación (Magisterio), Pedagogía y Educación Social del ámbito nacional}

Para continuar con nuestro estudio y responder a los objetivos planteados, se realiza un análisis exhaustivo de todos los planes de estudio de las titulaciones de Magisterio, Pedagogía y Educación Social de las universidades públicas, privadas y a distancia del ámbito nacional, con la finalidad de conocer si aparecen asignaturas relacionadas con la pedagogía hospitalaria. En la tabla 2 presentamos los datos referentes a la presencia de asignaturas relacionadas con la pedagogía hospitalaria en los grados de Magisterio de todas las universidades públicas del ámbito nacional que imparten los citados grados. 


\section{TABLA 2}

Universidades públicas del ámbito nacional que imparten asignaturas relacionadas con la pedagogía hospitalaria dentro de los grados de Magisterio

\begin{tabular}{|c|c|c|}
\hline Universidad & $\begin{array}{c}\text { Asignaturas relacionadas } \\
\text { con la } P . H .\end{array}$ & $\begin{array}{c}\text { Título de } \\
\text { la asignatura }\end{array}$ \\
\hline Universidad de A Coruña & No & - \\
\hline Universidad de Alcalá & No & - \\
\hline Universidad de Alicante & No & - \\
\hline Universitat Autònoma de Barcelona & No & - \\
\hline Universidad Autónoma de Madrid & No & - \\
\hline Universitat de Barcelona & No & - \\
\hline Universidad de Burgos & No & - \\
\hline Universidad de Cádiz & No & - \\
\hline Universidad de Cantabria & No & - \\
\hline Universidad Castilla-La Mancha & No & - \\
\hline Universidad Complutense de Madrid & No & - \\
\hline Universidad de Córdoba & No & - \\
\hline Universidad de Extremadura & No & - \\
\hline Universidad de La Rioja & No & - \\
\hline Universidad de Las Palmas & No & - \\
\hline Universidad de León & No & - \\
\hline Universitat de Lleida & No & - \\
\hline Universidad de Málaga & No & - \\
\hline Universidad de Murcia & No & - \\
\hline Universidad de Oviedo & No & - \\
\hline Universidad del País Vasco & No & - \\
\hline Universidad Pública de Navarra & No & - \\
\hline Universidad Rey Juan Carlos & No & - \\
\hline Universitat Rovira i Virgili & No & - \\
\hline Universidad de Salamanca & No & - \\
\hline Universidad Santiago Compostela & No & - \\
\hline Universidad de Sevilla & No & - \\
\hline Universitat de València & No & - \\
\hline Universidad de Valladolid & No & - \\
\hline
\end{tabular}




\begin{tabular}{|l|c|c|}
\hline \multicolumn{1}{|c|}{ Universidad } & $\begin{array}{c}\text { Asignaturas relacionadas } \\
\text { con la P. H. }\end{array}$ & $\begin{array}{c}\text { Título de } \\
\text { la asignatura }\end{array}$ \\
\hline Universidad de Vigo & No & - \\
\hline Universidad de Zaragoza & No & - \\
\hline
\end{tabular}

Fuente: Elaboración propia.

Como se puede observar en la tabla 2, no existe en los planes de estudio de los grados de Magisterio de ninguna universidad pública ninguna asignatura relacionada con la atención educativa al alumno enfermo.

Pasamos a presentar en la tabla 3 los datos referidos a la presencia de asignaturas relacionadas con la pedagogía hospitalaria en los grados de Magisterio de las universidades privadas, de la Iglesia católica y a distancia del ámbito nacional.

TABLA 3

Universidades privadas, de la Iglesia católica y a distancia del ámbito nacional que imparten asignaturas relacionadas con la pedagogía hospitalaria dentro de los grados de Magisterio

\begin{tabular}{|l|c|c|}
\hline \multicolumn{1}{|c|}{ Universidad } & $\begin{array}{c}\text { Asignaturas relacionadas } \\
\text { con la P. H. }\end{array}$ & $\begin{array}{c}\text { Título de } \\
\text { la asignatura }\end{array}$ \\
\hline Universitat Abat Oliba CEU & No & - \\
\hline Universidad Alfonso X El Sabio & No & - \\
\hline Universidad Antonio de Nebrija & No & - \\
\hline Universidad Camilo José Cela & No & - \\
\hline Universidad Cardenal Herrera-CEU & No & - \\
\hline Universidad Católica de Ávila & No & - \\
\hline Universidad Católica de Valencia & No & - \\
\hline Universidad Católica San Antonio & No & - \\
\hline Universidad de Deusto & No & - \\
\hline Universidad Europea de Madrid & No & - \\
\hline Universidad Europea de Valencia & No & - \\
\hline Universidad Francisco de Vitoria & No & - \\
\hline Universitat Internacional de Catalunya & No & - \\
\hline Universidad Loyola Andalucía & No & - \\
\hline
\end{tabular}




\begin{tabular}{|l|c|c|}
\hline \multicolumn{1}{|c|}{ Universidad } & $\begin{array}{c}\text { Asignaturas relacionadas } \\
\text { con la P. H. }\end{array}$ & $\begin{array}{c}\text { Título de } \\
\text { la asignatura }\end{array}$ \\
\hline Universidad Mondragón & No & - \\
\hline Universidad de Navarra & Sí & $\begin{array}{c}\text { Pedagogía Hospita- } \\
\text { laria }\end{array}$ \\
\hline Universidad Pontificia Comillas & No & - \\
\hline Universidad Pontificia de Salamanca & No & - \\
\hline Universitat Ramón Llull & No & - \\
\hline Universidad San Pablo-CEU & No & - \\
\hline Universitat de Vic & No & - \\
\hline Universidad a Distancia de Madrid & No & - \\
\hline Universidad Internacional de la Rioja & No & - \\
\hline Universidad Isabel I de Castilla & No & No \\
\hline Universitat Internacional Valenciana & & No \\
\hline
\end{tabular}

Fuente: Elaboración propia.

Una vez analizados los planes de estudios de todas las titulaciones de Magisterio de las universidades privadas, de la Iglesia católica y a distancia del ámbito nacional, en la tabla 3 observamos que es la Universidad de Navarra la única que oferta la asignatura de Pedagogía Hospitalaria dentro de los grados de Educación Infantil y Primaria. La asignatura tiene una carga de tres créditos y pertenece al Departamento de Teoría y métodos de investigación educativa y Psicológica de la Facultad de Psicología y Educación.

Destacamos que la Universidad de Vigo ofertó la asignatura "Educación y Hospital" dentro de especialidad de Educación Especial, convirtiéndose en la primera universidad de Europa que incorporó en sus planes de estudio la asignatura de Pedagogía Hospitalaria, la cual empezó teniendo una carga de cuatro créditos hasta llegar a los seis posteriormente. La asignatura llegó a tener a más de 200 alumnos matriculados. Incluso hubo alumnos de Enfermería que la cursaron como créditos de libre elección, antes de la implantación del Plan Bolonia. La Universidad de Vigo implantó créditos prácticos dentro de la formación en pedagogía hospitalaria, que contemplaba la realización del Practicum I y Practicum II en alguna de las escuelas hospitalarias de Galicia. La asignatura de Educación y Hospital también 
contemplaba una parte práctica, la cual se basaba en la realización de prácticas grupales en las aulas hospitalarias de los hospitales de Santiago de Compostela u Ourense.

Partiendo de los ejemplos anteriores, destacamos la viabilidad de la implantación de la pedagogía hospitalaria en los grados de Educación y la necesidad de ahondar en la importancia de la formación específica de los futuros docentes, ya que a los maestros hay primero que formarlos para después poder exigirles.

Siguiendo con la presentación de las universidades que imparten asignaturas relacionadas con la pedagogía hospitalaria dentro de los grados de Pedagogía, presentamos las tablas 4 y 5 , las cuales recogen los datos relativos tanto a las universidades públicas como privadas, a distancia y de la Iglesia católica que imparten el citado grado.

\section{TABLA 4}

Universidades públicas del ámbito nacional que imparten asignaturas relacionadas con la pedagogía hospitalaria dentro de los grados de Pedagogía

\begin{tabular}{|l|c|c|}
\hline Universidad & $\begin{array}{c}\text { Asignaturas relacionadas } \\
\text { con la P. H. }\end{array}$ & Título de la asignatura \\
\hline Universitat Autònoma de Barcelona & No & - \\
\hline Universitat de Barcelona & No & $\begin{array}{c}\text { Orientación en situaciones espe- } \\
\text { ciales: Atención hospitalaria y } \\
\text { menores en riesgo social }\end{array}$ \\
\hline Universidad de Burgos & Sí & - \\
\hline Universidad Complutense Madrid & No & - \\
\hline Universitat de Girona & No & - \\
\hline Universidad de Granada & No & - \\
\hline Universitat de les Illes Balears & No & - \\
\hline Universidad de La Laguna & No & - \\
\hline Universidad de Málaga & No & - \\
\hline Universidad de Murcia & No & - \\
\hline Universidad de Oviedo & No & - \\
\hline Universidad del País Vasco & No & - \\
\hline Universitat Rovira i Virgili & No & No \\
\hline Universidad de Salamanca & & - \\
\hline
\end{tabular}




\begin{tabular}{|l|c|c|}
\hline Universidad & $\begin{array}{c}\text { Asignaturas relacionadas } \\
\text { con la P. H. }\end{array}$ & Título de la asignatura \\
\hline Universidad de Santiago Compostela & Sí & Pedagogía Hospitalaria \\
\hline Universidad de Sevilla & No & - \\
\hline Universitat de València & No & - \\
\hline
\end{tabular}

Fuente: Elaboración propia.

Tras analizar los planes de estudio de todas las titulaciones de Pedagogía de las universidades públicas del ámbito nacional nos encontramos con la Universidad de Burgos, que incorpora en sus planes de estudio de Pedagogía una asignatura de carácter obligatorio titulada Orientación en situaciones especiales: Atención hospitalaria y menores en riesgo social. La asignatura tiene seis créditos, se imparte en el tercer curso y pertenece al Departamento de Ciencias de la Educación. La Universidad de Santiago de Compostela también oferta la asignatura "Pedagogía Hospitalaria". En este caso, la asignatura cuenta con 4,5 créditos y es optativa. Pertenece al Departamento de Didáctica y Organización Escolar, Métodos de Investigación y Diagnóstico en Educación de la Facultad de Ciencias de la Educación. Atendiendo a las universidades privadas, de la Iglesia católica y a distancia del ámbito nacional que imparten asignaturas relacionadas con la pedagogía hospitalaria dentro de los grados de Pedagogía, presentamos en la tabla 5 los datos extraídos del análisis documental.

TABLA 5

Universidades privadas, de la Iglesia católica y a distancia del ámbito nacional que imparten asignaturas relacionadas con la pedagogía hospitalaria dentro de los grados de Pedagogía

\begin{tabular}{|l|c|c|}
\hline \multicolumn{1}{|c|}{ Universidad } & $\begin{array}{c}\text { Asignaturas relacionadas } \\
\text { con la P. H. }\end{array}$ & Título de la asignatura \\
\hline Universidad de Navarra & Sí & Pedagogía Hospitalaria \\
\hline Universidad Pontificia de Salamanca & No & - \\
\hline UNED & No & - \\
\hline
\end{tabular}

Fuente: Elaboración propia. 
Como podemos observar en la tabla 5, la Universidad de Navarra oferta la asignatura de Pedagogía Hospitalaria con carácter optativo y con una carga de tres créditos.

A continuación presentamos las tablas 6 y 7, donde se recogen los datos referidos a las universidades del ámbito nacional que imparten asignaturas relacionadas con la pedagogía hospitalaria dentro de los grados de Educación Social.

\section{TABLA 6}

Universidades públicas del ámbito nacional que imparten asignaturas relacionadas con la pedagogía hospitalaria dentro de los grados de Educación Social

\begin{tabular}{|l|l|l|}
\hline \multicolumn{1}{|c|}{ Universidad } & $\begin{array}{c}\text { Asignaturas relacionadas } \\
\text { con la P. H. }\end{array}$ & Título de la asignatura \\
\hline Universidad de A Coruña & No & - \\
\hline Universidad de Alcalá & No & - \\
\hline Universitat Autònoma de Barcelona & No & - \\
\hline Universidad de Almería & No & - \\
\hline Universidad Autónoma de Madrid & No & - \\
\hline Universitat de Barcelona & No & - \\
\hline Universidad de Burgos & No & - \\
\hline Universidad de Castilla-La Mancha & No & - \\
\hline Universidad Complutense de Madrid & No & - \\
\hline Universidad de Córdoba & No & - \\
\hline Universidad de Extremadura & No & - \\
\hline Universitat de Girona & No & - \\
\hline Universidad de Granada & No & - \\
\hline Universidad de Huelva & No & - \\
\hline Universitat de les Illes Balears & No & - \\
\hline Universidad de Jaén & No & - \\
\hline Universidad de Las Palmas & No & - \\
\hline Universidad de León & No & - \\
\hline Universitat de Lleida & No & No \\
\hline Universidad de Málaga & No & - \\
\hline Universidad de Murcia & & - \\
\hline
\end{tabular}




\begin{tabular}{|l|c|c|}
\hline \multicolumn{1}{|c|}{ Universidad } & $\begin{array}{c}\text { Asignaturas relacionadas } \\
\text { con la P. H. }\end{array}$ & Título de la asignatura \\
\hline Universidad de Oviedo & No & - \\
\hline Universidad Pablo de Olavide & No & - \\
\hline Universidad del País Vasco & No & - \\
\hline Universitat Rovira i Virgili & No & - \\
\hline Universidad de Salamanca & No & - \\
\hline Universidad de Santiago de Compostela & No & Educación para la salud y \\
conductas adictivas
\end{tabular}

Fuente: Elaboración propia.

Como se puede observar en la tabla 6 se ha encontrado dentro de los grados de Educación Social de la Universidad de Valencia la asignatura "Educación para la Salud y conductas adictivas", la cual se oferta como asignatura optativa de $4 .^{\circ}$ curso. A continuación, pasamos a presentar en la tabla 7 los datos de la prevalencia de la pedagogía hospitalaria en las universidades privadas, de la Iglesia católica y a distancia del ámbito nacional que imparten los grados de Educación Social.

\section{TABLA 7}

Universidades privadas, de la Iglesia católica y a distancia del ámbito nacional que imparten asignaturas relacionadas con la pedagogía hospitalaria dentro de los grados de Educación Social

\begin{tabular}{|l|c|c|}
\hline \multicolumn{1}{|c|}{ Universidad } & $\begin{array}{c}\text { Asignaturas relacionadas } \\
\text { con la P. H. }\end{array}$ & $\begin{array}{c}\text { Título de } \\
\text { la asignatura }\end{array}$ \\
\hline Universidad Camilo José Cela & No & - \\
\hline Universidad Católica de Valencia San Vicente Mártir & No & - \\
\hline Universidad de Deusto & No & - \\
\hline Universidad Pontificia de Salamanca & No & - \\
\hline Universitat Ramón Llull & No & - \\
\hline Universitat de Vic & No & - \\
\hline
\end{tabular}




\begin{tabular}{|l|c|c|}
\hline \multicolumn{1}{|c|}{ Universidad } & $\begin{array}{c}\text { Asignaturas relacionadas } \\
\text { con la P. H. }\end{array}$ & $\begin{array}{c}\text { Título de } \\
\text { la asignatura }\end{array}$ \\
\hline Nacional de Educación a Distancia & No & - \\
\hline Universitat Oberta de Catalunya & No & - \\
\hline
\end{tabular}

Fuente: Elaboración propia.

Para finalizar con el estudio de la consideración actual de la pedagogía hospitalaria en las universidades, hemos procedido a investigar si existen posgrados específicos en esta materia en las universidades españolas. Resumimos esta información en la tabla 8 .

TABLA 8

Universidades del ámbito nacional que imparten posgrados relacionados con la pedagogía hospitalaria

\begin{tabular}{|l|l|c|}
\hline \multicolumn{1}{|c|}{ Universidad } & \multicolumn{1}{|c|}{ Título del máster } & Créditos \\
\hline Universidad de Barcelona & $\begin{array}{l}\text { Máster en atención integral desde la Pedagogía Hos- } \\
\text { pitalaria a lo largo de la vida }\end{array}$ & 60 \\
\hline Universidad de la Laguna & $\begin{array}{l}\text { Título propio experto universitario en una de las TIC } \\
\text { para la atención educativa hospitalaria y domiciliaria }\end{array}$ & 15 \\
\hline Universidad de Barcelona & $\begin{array}{l}\text { Diploma de Especialización /Posgrado } \\
\text { Pedagogía Hospitalaria en Neonatología y Pediatría }\end{array}$ & 33 \\
\hline Universitat Ramón Llull & $\begin{array}{l}\text { Diploma de especialización universitaria en Pedago- } \\
\text { gía Hospitalaria }\end{array}$ & 33 \\
\hline
\end{tabular}

Fuente: Elaboración propia.

Como podemos observar en la tabla 8 , en todo el ámbito nacional únicamente existen cuatro posgrados relacionados con la formación específica en pedagogía hospitalaria. Una vez aportados los resultados de nuestra investigación, pasamos a presentar en el siguiente apartado la discusión y conclusiones de nuestro trabajo. 


\section{DiSCUSIÓN Y CONCLUSIONES}

Aunque son muchos los avances que las investigaciones en materia de pedagogía hospitalaria han aportado a la disciplina, entre ellas las realizadas por autoras como Lizasoáin y Ochoa (2016) han puesto de manifiesto la escasa presencia de la pedagogía hospitalaria en la formación universitaria de los futuros profesionales de la Educación.

Ejemplo de los avances que en el campo de la pedagogía hospitalaria se van materializando son la ampliación del ámbito de acción de la citada disciplina mediante la intervención psicopedagógica en alumnos con necesidades específicas de apoyo educativo y la orientación vocacional y educativa de jóvenes con trastornos del neurodesarrollo y dificultades de aprendizaje derivados de la consulta de neuropediatría atendidos en la Unidad de Pedagogía Hospitalaria de la Clínica Universidad de Navarra (Lizasoáin y Ochoa, 2016).

Siguiendo con el apartado que nos ocupa, atendiendo al primer objetivo específico planteado, hemos expuesto que, durante el año 2016, fueron ingresados en los hospitales de ámbito nacional un total de 568.958 niños/as y jóvenes entre 0 y 24 años. La estancia media de estos ingresos se situó en 50 días. Conocer estos datos nos permite poner de manifiesto que el elevado número de niños/as que se ven alejados por un periodo de tiempo de su colegio a causa de la aparición de una enfermedad bien merece ser tenido en cuenta para que los/as profesionales de la Educación que atienden a estos/as menores, tanto en el hospital como en sus centros de referencia, velen por una atención educativa con las mayores garantías. Ello pasa por recibir una formación específica que ayude a paliar las consecuencias negativas y las alteraciones derivadas de la enfermedad. En concordancia con los datos reflejados en el presente artículo, encontramos entre otros autores las aportaciones de Lieutenant (2006), Ochoa (2015) o Lizasoáin (2016a), quienes insisten en la importancia de la formación de los docentes hospitalarios durante sus estudios universitarios para poder adaptarse a un contexto en continuo cambio.

Respecto al segundo objetivo específico propuesto en la investigación, centrado en conocer cómo se regula en nuestro país, y más concretamente en la Comunidad Valenciana, la atención educativa de los menores hospitalizados, hemos verificado que son los funcionarios pertenecientes al cuerpo 
de maestros y profesores de enseñanza secundaria a quienes se les encarga la tarea de ocupar los puestos de docente en las unidades pedagógicas hospitalarias. Tal y como se recoge en otros estudios, aunque la legislación vigente encarga a la Administración la tarea de formar a los maestros hospitalarios en pedagogía hospitalaria una vez que se ha accedido al mencionado puesto de trabajo, abogamos porque esa formación no esté dirigida exclusivamente al citado docente, sino a todos/as los/as profesionales de la educación que están implicados/as en la atención educativa del menor enfermo (Ruiz, 2016).

Por ello, defendemos que las vías más eficaces para reivindicar la necesidad de formación de los profesionales de la Educación en Pedagogía Hospitalaria son, por una parte, el aumento de investigaciones que pongan de manifiesto las necesidades psicoeducativas derivadas de la aparición de la enfermedad infantil, y, por otra, plantear la necesidad de implantar la asignatura de Pedagogía Hospitalaria en los grados de Educación.

Las afirmaciones anteriores se sustentan en los datos referentes a la forma de provisión de las vacantes en las unidades de pedagogía hospitalaria y que han sido presentados en el artículo que nos ocupa. A este respecto, aunque la mayoría de los maestros hospitalarios cuentan con la especialidad en pedagogía terapéutica, desde la implantación del Plan Bolonia las especialidades han desaparecido de los planes de estudio de los grados de Educación, pudiendo cursar exclusivamente los grados en Educación o Educación Primaria. Tal y como se afirma en otros trabajos, "esta formación en pedagogía terapéutica ha desaparecido, relegándose en el mejor de los casos a la posibilidad de estudiar una oposición centrada en la Educación Especial" (Ruiz, 2016: 322).

Las circunstancias que envuelven al niño enfermo en el hospital distan mucho de las circunstancias que rodean a un niño sano, y por tanto la acción educativa que sobre los niños enfermos se puede aplicar también requiere de unas metodologías específicas, basadas en cuestiones clave que la pedagogía Hospitalaria puede aportar. No debemos obviar que cuando un niño es diagnosticado con una enfermedad grave, toda la familia se enfrenta a un trance extremadamente estresante y a un profundo cambio en la vida familiar (Houtzager, Grootenhuis y Hoekstra-Weebers, 2005; Violant y Canet, 2015; Ruiz, 2016).

Autores como Álvarez y Del Río (2013) defienden la necesidad de una neuropsicología del desarrollo aplicada a la generalidad de la población in- 
fantil y el contexto hospitalario no debe permanecer al margen. En esta línea encontramos a Ochoa (2003) citada en Ochoa (2016), quien anticipaba que son necesarias las unidades de intervención psicopedagógicas en los contextos hospitalarios.

Considerando en este punto el tercer objetivo específico, afirmamos que realizar un análisis exhaustivo de prevalencia de la pedagogía hospitalaria en los actuales planes de estudio de los grados de Pedagogía, Educación Social y Magisterio sustenta la defensa de nuestra propuesta desde el profundo conocimiento de las mencionadas titulaciones.

Las ideas expuestas coinciden con las aportaciones de Doval y Estévez (2001), quienes indican que la especialidad de pedagogía hospitalaria es inexistente en los planes de formación universitarios europeos. Las autoras coinciden con Violant (2015b) y Fernández (2000) en la insuficiencia de las actuaciones que existen actualmente en España en relación con la formación en pedagogía hospitalaria. Estas actuaciones quedan reducidas a un mínimo porcentaje de universidades que han incluido en sus planes de estudio asignaturas optativas que abarcan esta temática. En el mejor de los casos existen escasas experiencias de formación llevadas a cabo por la Administración educativa. En palabras de las propias Doval y Estévez "la situación de la formación del profesor hospitalario es bastante preocupante" (2001: 15).

Por todo lo expuesto hasta el momento, concluimos nuestro trabajo con la pretensión de que la implementación de la formación de los futuros docentes y pedagogos en pedagogía hospitalaria constituya un paso para la mejora de la calidad de vida de los menores hospitalizados. Defendemos que todo docente debería tener unas competencias específicas en pedagogía hospitalaria adquiridas durante sus estudios universitarios que fueran implementadas por cursos de formación específicos o posgrados en el caso de desarrollar su carrera profesional dentro del contexto hospitalario y consideramos que dotar a la pedagogía hospitalaria de un corpus científico cada vez más sólido, gracias al trabajo e investigaciones de cuantos compañeros/as trabajan en esta línea, es el primer paso hacia la consecución de un objetivo común: que los niños enfermos sean atendidos educativamente por profesionales formados específicamente para ello, velando de este modo por un derecho fundamental de los/as menores: el derecho a la educación. 


\section{BiBLIOGRAFÍA}

Álvarez, A. y Del Río, P. (2013). El papel de la educación en el desarrollo: de la escuela a la cultura. Cultura y Educación, 25 (2), 137-151.

Anguera, M.T. y Violant, O. (2015). Investigación en Pedagogía Hospitalaria. Málaga: Aljibe.

Doval, I. y Estévez, N. (2001). Formación para la Consecución de competencias en Pedagogía Hospitalaria. La propuesta de la Universidad de Vigo desde el Prácticum y las prácticas de Educación y Hospital. En M. A. Zabalza et al. (coords.), El Practicum y las prácticas en empresas en la formación universitaria. Simposio llevado a cabo en el Simposium Internacional Sobre el Practicum, Pontevedra.

Fernández, M. (2000). La Pedagogía Hospitalaria y el Pedagogo Hospitalario. Tabanque, 15, 139-149.

González, F. E.; Macías, E. y García, F. (2002). La Pedagogía Hospitalaria: reconsideraciones desde la actividad educativa. Revista Complutense de Educación, 12(1), 303-365.

Houtzager, B. A.; Grootenhuis, M. A. y Hoekstra-Weebers, J. (2005). One month after diagnosis: quality of life, coping and previous functioning in siblings of children with cancer. Child Care Health and Development, 31(1), 75-87.

Instituto Nacional de Estadística (25/05/2018). Encuesta de morbilidad hospitalaria 2016 [Base de datos]. Recuperado de: https://www.ine.es/ dynt3/inebase/index.htm?padre $=4615$.

Instrucción de 18 de agosto de 2017, de la Dirección general de política educativa por la que se regula el funcionamiento de las Unidades Pedagógicas Hospitalarias ubicadas en hospitales públicos de la Comunidad Valenciana para el curso 2017/2018.

Lieutenant, C. (2006). La evolución de las aulas hospitalarias. Un camino por recorrer. En S. Riquelme (presidencia). Aulas Hospitalarias. Reflexiones sobre la VIII Jornada de Pedagogía Hospitalaria. Chile: Fundación Carolina Labra Riquelme.

LizasoÁrn, O. (2016a). Pedagogía Hospitalaria. Guía para la atención psicoeducativa del alumno enfermo. Madrid, España: Síntesis. 
LizasoÁin, O. (2016b). La Pedagogía Hospitalaria como un punto de partida para la orientación de alumnos con trastornos del neurodesarrollo. En O. Lizasoáin, B. Ochoa, A. Arellano, N. Crespo, S. Magallón y R. SánchezCarpintero, Alumnos con trastornos del neurodesarrollo. Pautas para su orientación educativa y vocacional desde la Pedagogía Hospitalaria (pp. 17-36). Pamplona: Ediciones Eunate.

LizasoÁin, O. y Lieutenant, C. (2002). La Pedagogía Hospitalaria frente a un niño con pronóstico fatal. Reflexiones en torno a la necesidad de una formación profesional específica. Estudios sobre Educación, 2, 157-165.

Lizasóín, O. y OchoA, B. (2016). Planteamiento de un proyecto de intervención centrado en la orientación vocacional para alumnos con trastornos del neurodesarrollo. En O. Lizasoáin, B. Ochoa, A. Arellano, N. Crespo, S. Magallón y R. Sánchez-Carpintero, Alumnos con trastornos del neurodesarrollo. Pautas para su orientación educativa y vocacional desde la Pedagogía Hospitalaria (pp. 37-60). Pamplona: Ediciones Eunate.

Ministerio de Educación, Cultura y Deporte (2018). QUEDU. Qué estudiar y dónde en la Universidad. Madrid: MECD. Recuperado de: https://www. educacion.gob.es/notasdecorte/compBdDo.

OchoA, B. (2015). Bases Conceptuales de la Pedagogía Hospitalaria. En C. González y V. Violant (comps.), Uso de las TIC para la atención educativa hospitalaria y domiciliaria (pp. 9-26). Madrid: Ediciones Universitarias Mc Graw-Hill.

OchoA, B. (2016). Orientación vocacional para alumnos con trastornos del neurodesarrollo. En O. Lizasoáin, B. Ochoa, A. Arellano, N. Crespo, S. Magallón y R. Sánchez-Carpintero, Alumnos con trastornos del neurodesarrollo. Pautas para su orientación educativa y vocacional desde la Pedagogía Hospitalaria (pp. 161-182). Pamplona: Ediciones Eunate.

Orden de 4 de julio de 2001, de la Consellería de Cultura y Educación por la que se regula la atención al alumnado con necesidades de compensación educativa. DOGV, 17 de junio de 2001, n. ${ }^{\circ}$ 4.044, pp. 19.282-16.293.

Orden de 21 de noviembre de 2006 de la Consellería de Cultura, Educación y Deporte, por la que determinan los criterios y procedimientos para la atención hospitalaria y domiciliaria del alumnado que requiera compensación educativa en educación primaria y secundaria obligatoria. DOGV, 26 de diciembre de 2006, n. ${ }^{\circ} 5.414$, pp. 4.674-4679. 
Ruzz, M. (2016). Pedagogía Hospitalaria. Hacia el diseño de una propuesta de competencias especificas para la formación docente. Tesis doctoral inédita. Facultad de Filosofía y Ciencias de la educación. Departamento de Teoría de la Educación. Universidad de Valencia: Valencia.

Torralba, F.; Torralba, F.; López, A. y Moreau, P. (2006). Educar en un mundo vulnerable. Lleida: Milenio.

Violant, V. (2015a). Competencias en el ejercicio profesional en Pedagogía Hospitalaria. En M. Alguacil (coord.), Perfil competencial del profesional que interviene durante la enfermedad y la convalecencia (pp. 15-45). Málaga: Aljibe.

Violant, V. (2015b). Competencias del perfil profesional implicado en la atención educativa en situación de enfermedad y hospitalización. En J. González (coord.), Memorias I Congreso Internacional de Pedagogía Hospitalaria. Congreso llevado a cabo en Bogotá, Colombia.

Violant, O. y CANET, O. (2015). Impacto de la enfermedad y la hospitalización. En O. Violant (coord.), Bases de la Pedagogía Hospitalaria aplicadas a las etapas vitales. Málaga: Aljibe.

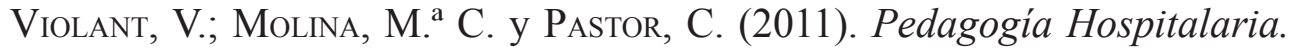
Bases para la atención integral. Barcelona: Laertes. 